\title{
Facts about Endosalpingiosis
}

\author{
Min Hou', Lipai Chen², Jingping Yun ${ }^{3 *}$ \\ ${ }^{1}$ Department of Pathology, Cancer Center, Guangzhou Medical University, Guangzhou, China \\ ${ }^{2}$ Department of Gynecological Oncology, Cancer Center, Guangzhou Medical University, Guangzhou, China \\ ${ }^{3}$ State Key Laboratory of Oncology in South China and Department of Pathology, Sun Yat-sen University Cancer \\ Center, Guangzhou, China \\ Email: *yunjp@sysucc.org.cn
}

Received 2 June 2015; accepted 29 August 2015; published 1 September 2015

Copyright (C) 2015 by authors and Scientific Research Publishing Inc.

This work is licensed under the Creative Commons Attribution International License (CC BY).

http://creativecommons.org/licenses/by/4.0/

c. (i) Open Access

\begin{abstract}
Endosalpingiosis was first described by Sampson in 1930. However, until recently more and more studies show the convincing evidence that it most probably originates from tubal cells. It has a close relationship with the development of serous tumors, especially low-grade serous carcinoma. The lesion does cause symptoms and signs, such as chronic abdominal pain or tumor-like mass, though it is often found accidently for other gynecologic problems. Occasionally atypical endosalpingiosis needs to be differentiated from a malignancy when it appears in an unusual site with worrying morphologic presentations or under some special circumstances. Owing to the facts that it may evolve quietly and continuously towards serous tumors, and that the lesions at different sites may evolve independently, the outcomes of its evolution can eventually kill the patient. Therefore, a proper recognition of the lesion will translate it into an adequate care for those patients. This review summarizes the most recent findings and makes thoughtful comments.
\end{abstract}

\section{Keywords}

Endosalpingiosis, Chronic Abdominal Pain, Serous Tumors, Tubal-Cell Origin, Carcinogenesis

\section{Introduction}

Endosalpingiosis is defined as ectopic presence of epithelial inclusions that lining cells resemble tubal epithelium in both morphology and immunophenotypes. The tubal epithelium consists of ciliated, unciliated columnar and intercalated cells. Some lymphocytes, shown by some studies as T cells [1] [2], are scattered just above the basement membrane. Though growing ectopically endosalpingiosis keeps the morphologic features of tubal epithelium. There are no endometrial stromal cells around the inclusions. Most often there is no or little hemorrhage accompanied the lesion [3]. The morphologic features of the lesion are used to differentiate endosalpingi-

*Corresponding author. 
osis from endometriosis under microscope.

Cilia on the apical side of tubal epithelium are a sign of cell maturation and differentiation, compatible with the function of the epithelium. When lining cells of endosalpingiosis undergo degeneration or inclusions are filled with secretions, they become flat and cilia are hardly seen. Unciliated cells are regarded as secretory cells, which are observed to expand in cell proliferation or with age [1] [4] (reviewed in [5] [6]). Immunohistochemisty can be applied to determine the cell origin of the inclusions. PAX-8 and calretinin are the most useful markers [1] [6]. Tubulin can show cilia on the apical surface of the epithelium [1] [6].

Endosalpingiosis was first described by Sampson in 1930. The ovary is the most common site though the lesion has been reported to appear in many tissues and organs both inside and outside pelvic cavity [7]-[12]. The lining cells of the inclusions may undergo proliferation, which exhibits as multilayers of cells, cell clusters, papillary structure, and even free papillae [3] [13] (also personal observations). Occasionally the proliferation is profound and accompanied with cellular atypia, a condition defined as atypical endosalpingiosis [13]. Psammoma-body like calcification can appear on site [3] [14].

In this review we are going to discuss the topics including histogenesis of endosalpingiosis, its symptoms and signs, and relationship with the development of serous tumors. More importantly we are going to make some thoughtful comments in several aspects that are related to both pathologic diagnosis and patient care, based on our broad reading and working experiences.

\section{Histogenesis of Endosalpingiosis}

Several theories about the development or origination of endosalpingiosis have been proposed in the publications. Among them two are more popular. One is as secondary Mullerian origin that considers either the invagination of coelomic cells into the tissues or the rests of such cells from fetal development go through Mullerian metaplasia to form endosalpingosis [3]. The theory is purely postulated without direct observations to support.

The alternative theory, based on numerous observations and laboratory investigations, is tubal-cell origin [1] [6] [14]. In support of the theory is the intense proliferation of tubal cells, which presents as cell tufting or papillae with narrow stalks at the base; or sometimes as piles of cells with poor adhesion [6]. Such forms of tubal-cell proliferation give rise to the possibility that papillae are detached from the tubal epithelium by breaking narrow stalks or as a cluster of poorly-adhesive cells [6]. Then, freed tubal cells are shed and seeded in any tissues and organs in the pelvic cavity. The slow growth of seeded cells may develop into ectopic epithelial inclusions [10]. The theory of tubal cell origin is plausible in that it is able to explain why the lesion is exclusively seen in women [14]; and why the lesion is most frequently seen in the ovaries [15].

As to what to cause tubal cells to proliferate, shed and seed, the underlying mechanisms are poorly understood. Chronic tubal inflammation is considered to contribute to the process [6] [14]. The expansion of secretory cells with age [4] provides some evidence of genetic factors that will affect the susceptibility of tubal cells to various stimuli. Thus, the individual genetic composition imprinted in the endosalpingiosis will reasonably influence its evolution and outcome.

\section{Symptoms and Signs of Endosalpingiosis}

Most often the lesion exhibits few symptoms and signs and is accidently found for other gynecologic problems. However, it does cause chronic abdominal pain and tissue adhesion [16]. One meticulous study shows that $40 \%$ of the endosalpingiosis occur in post-menopausal patients; and 34.5\% coexist with endometriosis [17], a condition probably makes the coexisting endometriosis refractory or partially responsive to hormonal therapy [16]. Endometriosis is significantly correlated with infertility and chronic abdominal pain; while endosalpingiosis is not [17]. Morphologically both endosalpingosis and endometriosis can cause tissue adhesion; however, the remote and recent hemorrhage is not observed on the site of endosalpingosis [3]. When inclusions of endosalpingiosis are large in number and/or cystic, the lesion may present as a tumor-like mass [18]-[20]. Occasionally atypical endosalpingiosis in lymph nodes or in rare sites outside pelvic cavity with or without calcifications may be mistaken as a malignancy, especially in frozen sections [21] [22].

We have observed that endosalpingosis in pelvic tissues displays a spectrum of morphologic changes, from atrophy, monolayer, and proliferation to aypia with psammoma-body like calcification (data not published yet). Interestingly, its clinical presentations also seem to display a spectrum of changes, from no or few symptoms and signs, a tumor-like mass to a serous tumor. Both clinical and morphologic variations may reflect the changes 
of ectopically growing tubal cells vary at molecular levels with each condition, which leads to different pathologic outcomes.

\section{Relationship with the Development of Serous Tumors}

A body of evidence show that endosalpingiosis is the precursor of serous tumors, especially low-grade serous carcinoma [1] [4] (reviewed in [5] [6]). Li and associates [1] claim that 78\% of the ovarian inclusions are of tubal-cell origin. The proliferative index is higher in lining cells of the inclusions than in those of the fimbriae. The morphologic and immunohistochemical phenotypes resemble more like ovarian cystadenoma and borderline serous tumor. In fact, transitional changes from hyperplasia, dysplasia to carcinoma can often be observed; and such tumorous transformation may happen in multiple foci that are at different stages [23]-[27]. For example, ovarian borderline serous tumors are observed with low-grade serous carcinoma in pelvic lymph nodes [23]. Molecular studies conclude that serous low malignant potential implants in the ovaries and lymph node inclusions may have different origins [28].

Endosalpingiosis is considered to have an association with the relapse of ovarian serous tumors [29] [30]. Silva and coworkers [29] report that 11 cases of stage I ovarian borderline serous tumors, treated with hystectomy and bilateral oophorectomy and followed up for 7 to 39 years (average 16), are found relapsed. Ten of the 11 get serous carcinoma; and one has borderline serous tumors. The recurred tumors are located mainly in pelvic and abdominal cavity (8 cases); other sites include neck (2 cases) but followed with the involvement of abdominal cavity, and pleura (1 case) without any signs of pelvic or abdominal involvement. When those 11 relapsed patients were compared with 15 un-relapsed patients matched with age and tumor type, the only significant factor is the higher incidences of endosalpingiosis ( $72.7 \%$ vs $12.5 \%)$ at the time of initial diagnosis. Similar findings are also described in another study but all cases involve extra-abdominal/extra-pelvic sites [30]. Since the tumors reoccur long after the radical excision of the primaries, the observations make one wondering whether the recurred tumors are actually the second primaries. The concurrent endosalpingiosis can be the novel place of tumor development [23]-[27].

\section{Comments}

Based on the tubal-cell theory and the way of its formation, most often endosalpingiosis is not solitary. Once the lesion is found in one site, there is a great possibility that it exists in other sites, especially the ovaries. Since endosalpingosis is hard to be defined before and during surgery unless it causes significant pathologic changes, such as adhesion, calcification or cyst, it is indeed a challenge for those who want to reserve the ovaries in dealing with their gynecologic problems. An accident post-surgery finding of the lesion may indicate some extra caution to the future care of the patient.

The diagnosis of endosalpingiosis will be made during conventional pathologic observations if a pathologist is aware of the lesion. Under tough circumstances immunohistochemical analysis is used for differential diagnoses. PAX-8 positive and calretinin negative indicate the tubal-cell origin. Sometimes tubulin is used to show cilia, which can help estimate the ratio of secretory over ciliated cells. In reality the lesion is sometimes overlooked because of its superficial benign morphology versus a major problem of gynecologic malignancies; or mistaken as metastatic foci if tumors in the ovaries are serous carcinomas (personal experience). Only on rare occasions it attracts some attention [18]-[20]. Based on its relationship with the development of serous tumors we feel that the morphologic presentations of the lining cells need to be addressed in details in routine pathologic reports.

At the moment the lines between ovarian serous cystadenoma and cystic endosalpingiosis is obscure. In daily practice a cyst in the ovary lined with cuboidal cells is logically diagnosed as a serous cystadenoma if large enough. Perhaps there is little necessity to differentiate the one from the other for they may be very close in nature [1]. However, one may face a dilemma when a cyst presents with focal papillary structure lined by atypical cells. Obviously a state as serous cystadenoma with focal borderline changes overrates the pathologic observations. In consideration of the continuous evolution of endosalpingiosis a detailed description about the morphologies of lining epithelial cells may better define the nature of the lesion and give clinicians a clearer picture about the lesion. After all, clinicians and pathologists have to reach a consensus about the situation in order to provide an adequate care for patients [22].

The facts gathered from broad publications and our own observations indicate that endosalpingiosis is by no 
means as simple as ectopic tubal-cell inclusions. In a long-term process of quiet and continuous evolution, the accumulation of mutations of key genes, such as KRAS, BRAF, PTEN and PIK3CA (reviewed in [31]), may transform a focus or foci of the lesion to a carcinoma that may eventually kill the patient. Some investigators argue that primary serous tumors in the ovaries and other pelvic tissues and organs, including in peritoneum, almost all originate, if not all, from tubal cells [15]. Here we have to leave high-grade ovarian serous carcinoma out for comments since its carcinogenesis is more complicated. The evolution of endosalpingiosis may also be influenced by the microenvironment of the lodging site, for example, patients of primary peritoneal serous tumors seem to be older than those of ovarian serous tumors and molecular changes are somewhat different from ovarian serous tumors (reviewed in [32]).

\section{Conclusion}

Endosalpingiosis is an independent entity both clinically and pathologically. Though right now the underlying mechanisms of its development are poorly understood, a body of evidence demonstrates that both chronic tubal inflammation and genetic compositions contribute to the proliferation, shedding and seeding of tubal cells. The recognition of the lesion lies in its quiet and continuous evolution that may lead to a dreadful outcome of serous carcinoma in addition to other problems. Accurate pathologic diagnosis and proper management of the patients to avoid overtreatment or substandard care are the main purposes achieved in this review.

\section{References}

[1] Li, J., Abushahin, N., Pang, S., Xiang, L., Chambers, S.K., Fadare, O., et al. (2011) Tubal Origin of “Ovarian” Low-Grade Serous Carcinoma. Modern Pathology, 24, 1488-1499. http://dx.doi.org/10.1038/modpathol.2011.106

[2] Morris, H., Emms, M., Visser, T. and Timme, A. (1986) Lymphoid Tissue of the Normal Fallopian Tube-A Form of Mucosal-Associated Lymphoid Tissue (MALT)? International Journal of Gynecological Pathology, 5, 11-22. http://dx.doi.org/10.1097/00004347-198603000-00002

[3] Horn, L.C. and Bilek, K. (1995) Frequency and Histogenesis of Pelvic Retroperitoneal Lymph Node Inclusions of the Female Genital Tract. An Immunohistochemical Study of 34 Cases. Pathology, Research and Practice, 191, 991-996. http://dx.doi.org/10.1016/S0344-0338(11)80597-2

[4] Li, J., Ning, Y., Abushahin, N., Yuan, Z., Wang, Y., Wang, Y., et al. (2013) Secretory Cell Expansion with Aging: Risk for Pelvic Serous Carcinogenesis. Gynecologic Oncology, 131, 555-560. http://dx.doi.org/10.1016/j.ygyno.2013.09.018

[5] Li, J., Fadare, O., Xiang, L., Kong, B. and Zheng, W. (2012) Ovarian Serous Carcinoma: Recent Concepts on Its Origin and Carcinogenesis. Journal of Hematology and Oncology, 5, 8. http://dx.doi.org/10.1186/1756-8722-5-8

[6] Kurman, R.J., Vang, R., Junge, J., Hannibal, C.G., Kjaer, S.K. and Shih, IeM. (2011) Papillary Tubal Hyperplasia: The Putative Precursor of Ovarian Atypical Proliferative (Borderline) Serous Tumors, Noninvasive Implants, and Endosalpingiosis. The American Journal of Surgical Pathology, 35, 1605-1614. http://dx.doi.org/10.1097/PAS.0b013e318229449f

[7] Stolnicu, S., Preda, O., Kinga, S., Marian, C., Nicolau, R., Andrei, S., et al. (2011) Florid, Papillary Endosalpingiosis of the Axillary Lymph Nodes. The Breast Journal, 17, 268-272. http://dx.doi.org/10.1111/j.1524-4741.2011.01081.x

[8] Patonay, B., Semer, D. and Hong, H. (2011) Florid Cystic Endosalpingiosis with Extensive Peritoneal Involvement and Concurrent Bilateral Ovarian Serous Cystadenoma. Journal of Obstetrics and Gynaecology, 31, 773-774. http://dx.doi.org/10.3109/01443615.2011.608865

[9] Rosenberg, P., Nappi, L., Santoro, A., Bufo, P. and Greco, P. (2011) Pelvic Mass-Like Florid Cystic Endosalpingiosis of the Uterus: A Case Report and a Review of Literature. Archives of Gynecology and Obstetrics, 283, 519-523. http://dx.doi.org/10.1007/s00404-010-1700-1

[10] Maniar, K.P., Kalir, T.L., Palese, M.A. and Unger, P.D. (2010) Endosalpingiosis of the Urinary Bladder: A Case of Probable Implantative Origin with Characterization of Benign Fallopian Tube Immunohistochemistry. International Journal of Surgical Pathology, 18, 381-383.

[11] Tanahashi, J., Kashima, K., Daa, T., Kondo, Y., Kitano, S. and Yokoyama, S. (2006) Florid Cystic Endosalpingiosis of the Spleen. Acta Pathologica, Microbiologica, et Immunologica Scandinavica, 114, 393-398. http://dx.doi.org/10.1111/j.1600-0463.2006.apm_388.x

[12] Redondo, P., Idoate, M. and Corella, C. (2001) Cutaneous Umbilical Endosalpingiosis with Severe Abdominal Pain. Journal of the European Academy of Dermatology and Venereology, 15, 179-180. http://dx.doi.org/10.1046/j.1468-3083.2001.00263.x 
[13] Horn, L.C. and Bilek, K. (1996) Atypical (Carcinoma-Like) and Simple Glandular Inclusions in Retroperitoneal Lymph Nodes. General and Diagnostic Pathology, 141, 319-325.

[14] Zinsser, K.R. and Wheeler, J.E. (1982) Endosalpingiosis in the Omentum: A Study of Autopsy and Surgical Material. The American Journal of Surgical Pathology, 6, 109-117. http://dx.doi.org/10.1097/00000478-198203000-00003

[15] Kurman, R.J. and Shih, IeM. (2010) The Origin and Pathogenesis of Epithelial Ovarian Cancer: A Proposed Unifying Theory. The American Journal of Surgical Pathology, 34, 433-443. http://dx.doi.org/10.1097/PAS.0b013e3181cf3d79

[16] deHoop, T.A., Mira, J. and Thomas, M.A. (1997) Endosalpingiosis and Chronic Pelvic Pain. The Journal of Reproductive Medicine, 42, 613-616.

[17] Prentice, L., Stewart, A., Mohiuddin, S. and Johnson, N.P. (2012) What Is Endosalpingiosis? Fertility and Sterility, 98, 942-947. http://dx.doi.org/10.1016/j.fertnstert.2012.06.039

[18] Singh, N., Murali, S. and Zangmo, R. (2014) Florid Cystic Endosalpingiosis, Masquerading as Malignancy in a Young Patient: A Brief Review. BMJ Case Reports, 2014, Published 30 January 2014. http://dx.doi.org/10.1136/bcr-2013-201645

[19] Partyka, L., Steinhoff, M. and Lourenco, A.P. (2014) Endosalpingiosis Presenting as Multiple Pelvic Masses. Journal of Obstetrics and Gynaecology, 34, 279-281. http://dx.doi.org/10.3109/01443615.2013.859239

[20] Liang, J.J., Malpica, A. and Broaddus, R.R. (2007) Florid Cystic Endosalpingiosis Presenting as an Obstructive Colon Mass Mimicking Malignancy: Case Report and Literature Review. Journal of Gastrointestinal Cancer, 38, 83-86. http://dx.doi.org/10.1007/s12029-008-9036-9

[21] Corben, A.D., Nehhozina, T., Garg, K., Vallejo, C.E. and Brogi, E. (2010) Endosalpingiosis in Axillary Lymph Nodes: A Possible Pitfall in the Staging of Patients with Breast Carcinoma. The American Journal of Surgical Pathology, 34, 1211-1216. http://dx.doi.org/10.1097/PAS.0b013e3181e5e03e

[22] Singhania, N., Janakiraman, N., Coslett, D. and Ahmad, N. (2014) Endosalpingiosis in Conjunction with Ovarian Serous Cystadenoma Mimicking Metastatic Ovarian Malignancy. The American Journal of Case Reports, 15, 361-363. http://dx.doi.org/10.12659/AJCR.890921

[23] Djordjevic, B. and Malpica, A. (2012) Ovarian Serous Tumors of Low Malignant Potential with Nodal Low-Grade Serous Carcinoma. The American Journal of Surgical Pathology, 36, 955-963. http://dx.doi.org/10.1097/PAS.0b013e31825793e1

[24] Djordjevic, B., Clement-Kruzel, S., Atkinson, N.E. and Malpica, A. (2010) Nodal Endosalpingiosis in Ovarian Serous Tumors of Low Malignant Potential with Lymph Node Involvement: A Case for a Precursor Lesion. The American Journal of Surgical Pathology, 34, 1442-1448. http://dx.doi.org/10.1097/PAS.0b013e3181f17d33

[25] McCluggage, W.G., O’Rourke, D., McElhenney, C. and Crooks, M. (2002) Mullerian Papilloma-Like Proliferation Arising in Cystic Pelvic Endosalpingiosis. Human Pathology, 33, 944-946. http://dx.doi.org/10.1053/hupa.2002.127437

[26] McCoubrey, A., Houghton, O., McCallion, K. and McCluggage, W.G. (2005) Serous Adenocarcinoma of the Sigmoid Mesentery Arising in Cystic Endosalpingiosis. Journal of Clinical Pathology, 58, 1221-1223. http://dx.doi.org/10.1136/jcp.2005.027946

[27] Prade, M., Spatz, A., Bentley, R., Duvillard, P., Bognel, C. and Robboy, S.J. (1995) Borderline and Malignant Serous Tumor Arising in Pelvic Lymph Nodes: Evidence of Origin in Benign Glandular Inclusions. International Journal of Gynecological Pathology, 14, 87-91. http://dx.doi.org/10.1097/00004347-199501000-00015

[28] Emerson, R.E., Wang, M., Liu, F., Lawrence, W.D., Abdul-Karim, F.W. and Cheng, L. (2007) Molecular Genetic Evidence of an Independent Origin of Serous Low Malignant Potential Implants and Lymph Node Inclusions. International Journal of Gynecological Pathology, 26, 387-394. http://dx.doi.org/10.1097/pgp.0b013e3180336287

[29] Silva, E.G., Tornos, C., Zhuang, Z., Merino, M.J. and Gershenson, D.M. (1998) Tumor Recurrence in Stage I Ovarian Serous Neoplasms of Low Malignant Potential. International Journal of Gynecological Pathology, 17, 1-6. http://dx.doi.org/10.1097/00004347-199801000-00001

[30] Malpica, A., Deavers, M.T., Gershenson, D., Tortolero-Luna, G. and Silva, E.G. (2001) Serous Tumors Involving Extra-Abdominal/Extra-Pelvic Sites after the Diagnosis of an Ovarian Serous Neoplasm of Low Malignant Potential. The American Journal of Surgical Pathology, 25, 988-996. http://dx.doi.org/10.1097/00000478-200108000-00002

[31] Gadducci, A., Guerrieri, M.E. and Genazzani, A.R. (2012) New Insights on the Pathogenesis of Ovarian Carcinoma: Molecular Basis and Clinical Implications. Gynecological Endocrinology, 28, 582-586. http://dx.doi.org/10.3109/09513590.2011.649595

[32] Pentheroudakis, G. and Pavlidis, N. (2010) Serous Papillary Peritoneal Carcinoma: Unknown Primary Tumour, Ovarian Cancer Counterpart or a Distinct Entity? A Systematic Review. Critical Reviews in Oncology/Hematology, 75, 27-42. http://dx.doi.org/10.1016/j.critrevonc.2009.10.003 